\title{
A REVIEW OF SLOW-AND FAST-LIGHT BASED ON STIMULATED BRILLOUIN SCATTERING IN FUTURE OPTICAL COMMUNICATION NETWORKS
}

Slow- and Fast-Light has been investigated during the last years as a powerful tool to reduce and increase the group velocity of light pulses by light. It has enjoyed much recent interest because of the practical applications for telecommunication and information systems. Among these are optical signal processing, radio frequency-photonics, nonlinear optics and time domain spectroscopy. Furthermore, it can be seen as a key technology for optical delay lines, buffers, equalizers and synchronizers in packet switched networks. For a realization, there are different methods and material systems possible. Among them the nonlinear effect of stimulated Brillouin scattering (SBS) is of special interest. This article gives an overview about the fundamentals and some experimental results of the Slow-and Fast-Light effect based on the SBS in optical fibers.

\section{Introduction}

Today's data networks consist of optical fibers for the transport of data signals and network nodes for their switching through the net. The data traffic is nearly doubled in transport networks every year. But, this is not a big problem for optical transmission technologies because every fiber is able to transmit more than 100 channels with data rates of 10 or $40 \mathrm{Gbit} / \mathrm{s}$. Contrary to this, the capacity of network nodes is doubled only every 18 months [1].

Inside every node the optical pulses are converted into electrical signals to process and switch them. After that they are converted back into the optical domain to transmit them to the next

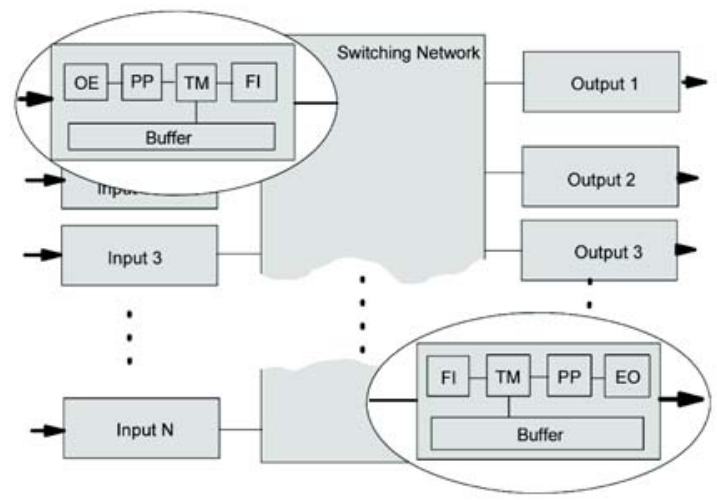

Fig. 1 Schematic setup of a network node. OE; optical-electrical conversion, PP; packet processor, TM; traffic manager, FI; fabric interface, EO; electrical-optical conversion [2]. node over the fiber. For the processing of the signal in the node it is necessary to store the pulses for a certain time otherwise collisions will occur. Therefore, every signal channel needs its own card for the handling which is shown in Fig. 1.

If the data traffic increases in the networks the number of channels and so the number of handling cards increases as well. Then, one problem is that the physical dimensions of the nodes expand. Hence, the distance between the in- and the output of the card increases drastically. Electrical signals with a high frequency cannot pass long distances without suffering significant losses and distortions. But, for optical signals this is not a problem. That is why there has began a reinforced development of optical alternatives to electrical network nodes. These optical nodes should take over all functions of the electrical ones including signal buffering.

Most functions of such an optical network node have already been shown [3], [4]. But, up to now the intermediate storage of the signals is not a satisfactorily solved problem. For an ideal processing the packets have to be buffered on the in- and outputs of the network channel cards. An optical buffer can be defined as follows [5]:

- The data stream is completely optically. No optical-electricaloptical conversion is proceeded.

- The buffer stores the signal for a time $\Delta T$ only with low distortions and attenuations.

- The delay time $\Delta T$ is variable and externally controllable.

The easiest way to realize an optical pulse delay is to send the signal into a delay line with a fixed length $L$, e. g. an optical fiber. Inside the fiber segment the signal propagates with the group

\footnotetext{
${ }^{*}$ Ronny Henker ${ }^{1}$, Andrzej Wiatrek ${ }^{1}$, Kai-Uwe Lauterbach ${ }^{1}$, Markus Junker ${ }^{1}$, Thomas Schneider ${ }^{1}$, Max J. Ammann ${ }^{2}$, Andreas T. Schwarzbacher ${ }^{2}$

${ }^{1}$ Hochschule für Telekommunikation Leipzig (FH), Leipzig, Germany, E-mail: ronny.henker@hft-leipzig.de

${ }^{2}$ School of Electronic \& Communications Engineering, Dublin Institute of Technology, Kevin Street Dublin 8, Ireland
} 
velocity $v_{g}$ which depends on the group index $n_{g}$ of the transmitting medium. If the waveguide dispersion is neglected it is:

$$
v_{g}=\frac{c}{n_{g}}
$$

with $c$ as the speed of light in vacuum. The time delay is caused by the fiber length and can be written as $\Delta T=a L / v_{g}$ with $a$ as the number of passes through the delay line. Hence, the storage capacity and the number of bits, respectively, depend on $\Delta T$. But, if the number of bits is longer interferences occur at the beginning and the end of the packet. And, if the packet is coupled into the fiber once it can be read out only after a whole circulation.

The main problem of this method is that the storage time is fixed and cannot be varied. But, in data networks the storage time has to be variable because the arrival time is stochastic and not quantized. Furthermore, the attenuation of the signal in long passive delay lines is another problem. Due to these disadvantages the socalled "Slow-Light" concept which could provide a way out of these problems has been discussed and developed for the last few years.

\section{Slow and Fast-Light}

For Slow- and Fast-Light the time delay does not depend on the length of a fiber but on the value of the group index $n_{g}$. According to Eq. (1) the optical signal can be slowed down (Slow-Light) or accelerated (Fast-Light) if it is possible to change $n_{g}$ along the propagation path. Thereby, the group index and the time delay depend on the frequency $\omega[2]$ :

$$
n_{g}=n(\omega)+\omega \frac{d n(\omega)}{d \omega},
$$

with $n$ as the real part of the refractive index of the material. Due to a positive change of the frequency dependence of the refractive index $\omega d n / d \omega$ the group index increases and hence the optical signals are delayed otherwise they are accelerated.
A large change of the group delay is caused automatically by a strong material dispersion. Mostly, such a dispersion occurs if the frequency of the light is nearby material resonances which results in absorption or amplification processes [2]. On the socalled Slow-Light effect these resonances are created artificially. For this, different methods and material systems can be used. The slowing down of light signals was shown for example in ultra cold [6] and hot atomic-gases [7], in semiconductor-nanostructures [5], quantum-well [8] and quantum-dot systems [9] as well as in waveguides which work like photonic crystals [10]. Furthermore, amplification effects like in Erbium-doped fiber amplifiers (EDFA) [11] and semiconductor amplifiers (SOA) [12] can also be used. All these methods have the disadvantage that the delay is very low and the effort is high. Some of these methods are also difficult to integrate into optical networks. In contrast to this, nonlinear effects in a fiber are more effective to delay optical signals. They have the great advantage that the fibers themselves are the Slow-light medium and can be integrated seamlessly in optical systems. For example four wave mixing together with the fiber dispersion [13] and Raman scattering [14] can be used to create Slow-Light. But the effect of SBS is of very special interest [15].

\section{Brillouin scattering}

The SBS has several advantages among the other Slow-Light methods. It needs just small pump powers for high time delays. The systems are very easy to implement and can be built up with standard components of telecommunications. Furthermore, the SBS works in all fiber types in their entire transparency range what makes the systems very flexible. In [16] it has been shown that the SBS can influence the group velocity over a wide range from $71000 \mathrm{~km} / \mathrm{s}$ to vacuum superluminal velocity.

The SBS is a nonlinear effect with a low threshold which is caused by interactions between the incident light and the material. The principle can be seen on the left hand side of Fig. 2. If a strong pump wave with the frequency $f_{p}$ is propagating through
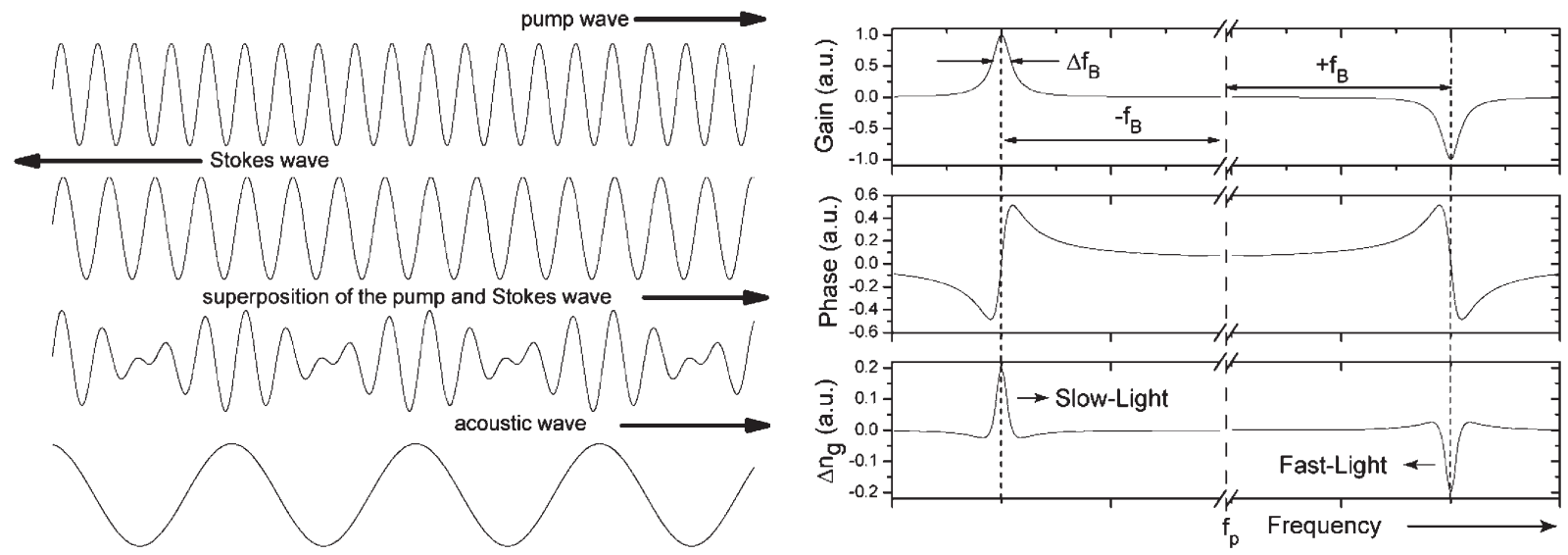

Fig. 2 Principle of SBS (left) [17]; Gain and loss (top), phase (middle) and group index change (bottom) via SBS (right) [2]. 
a waveguide a part of the optical power is backscattered on density fluctuations and a Stokes-wave occurs. This superimposes with the pump wave and creates a density wave in direction to the pump wave. There, more pump power is scattered and the process is built up. From a certain pump power, the Brillouin threshold, it becomes stimulated. Thereby, the pump and the density wave have a relative speed to each other, and hence the Stokes-wave is shifted in frequency by the so-called Brillouin shift $f_{B}$ which is around $11 \mathrm{GHz}$ in a standard single mode fiber (SSMF) at a pump wavelength of $1550 \mathrm{~nm}$ [17].

As can be seen in the upper diagram on the right hand side of Fig. 2 a gain and a loss is created in the fiber, if the input power is under the threshold. Thereby, the gain is downshifted and the loss is upshifted by $f_{B}$. Furthermore, they come along with a phase change and with a change of the group index (middle and bottom part on the right hand side of Fig. 2). Hence, within the gain and the loss a counter propagating signal wave can be amplified or attenuated. But, it can also be delayed or accelerated because, according to Eq. (2), the gradient of the group index is positive for $f_{p}-f_{B}$ and negative for $f_{p}+f_{B}$.

\section{Slow and Fast-Light based on SBS}

The output pulse $A(\omega, z)$ after the Slow-Light system relating to the input pulse $A(\omega, 0)$ is:

$$
A(\omega, z)=A(\omega, 0) e^{j k(\omega) z}
$$

with $z$ as the length of the medium and $k(\omega)$ as the complex wave number. The propagation of a pulse through the Slow-Light medium can be described by the complex wave number [17], [18]:

$$
k(\omega)=\sum_{i=0}^{\infty} \frac{k_{i}\left(\omega-\omega_{0}\right)^{i}}{i !} \text { with , } k_{i}=\left[\frac{d^{i} k}{d \omega^{i}}\right]_{\omega=\omega_{0}}
$$

where $\omega_{0}$ is the line center frequency. The first derivation $k_{1}=$ $=d k / d \omega$ corresponds to the reciprocal group velocity which leads to the transmission time of the signal whereas the second derivation $k_{2}=d^{2} k / d \omega^{2}$ is the group velocity dispersion (GVD) which leads to a distortion of the pulses. Without an influence of the SBS $k$ can be expressed by $k=\omega n_{0} / c$ with $n_{0}$ as the complex refractive index in the fiber. So, the time delay due to the propagation through the fiber is $\Delta T=z\left(k_{1}-1 / c\right)=z\left(n_{0}-1\right) / c$. For a single Brillouin gain with a Lorentzian distribution the complex wave number has to be expanded to [19]:

$$
k(\omega)=\frac{n_{0}}{c} \omega+\frac{g_{1}}{z}\left(\frac{\gamma_{1}}{\left(\omega-\omega_{0}\right)+j \gamma_{1}}\right),
$$

with $\gamma_{1}=\pi \Delta f_{B}$ as the half width at half maximum (HWHM) bandwidth of the gain and $g_{1}=g P L_{\text {eff }} / A_{\text {eff }}$ as the line center gain where $g$ is the Brillouin coefficient, $P$ is the pump power, $L_{\text {eff }}$ and $A_{\text {eff }}$ are the effective length and area of the fiber. The imaginary part of Eq. (5) leads to an amplification of the pulses and the real part to a phase change. Furthermore, the derivation of the real part leads to the group index change which causes the Slow-Light delay. Then, the time delay in the line center is $\Delta T=z\left(n_{0}-1\right) / c+$ $+g_{1} / \gamma_{1}$. Hence, the time delay only caused by the SBS can be written as:

$$
\Delta T_{S B S}=\frac{g_{1}}{\gamma_{1}} .
$$

As can be seen from Eq. (6), the time delay depends on the gain and on the pump power on the one hand and on the Brillouin bandwidth on the other hand. If not a Brillouin gain but a Brillouin loss is used $g_{1}$ and so the time delay becomes negative. Hence, the pulse is accelerated and Fast-Light is achieved.

A principle experimental setup for the generation of Slowand Fast-Light is shown on the left hand side of Fig. 3. A signal laser creates the carrier wave with the frequency $f_{s}$ for the pulses which are generated by a pulse generator combined with a MachZehnder modulator (MZM). Normally, we use Gaussian shaped pulses at wavelengths of around $1550 \mathrm{~nm}$. Then, they are coupled into a $50 \mathrm{~km}$ long SSMF which is our Slow- and Fast-Light medium. From the other side a pump wave (Pumplaser) with the frequency $f_{p}$ of a DFB-laser diode is coupled into the same fiber via an optical circulator (C). The pump wave creates a Brillouin gain at $f_{p}-f_{B}$ and a loss at $f_{p}+f_{B}$ inside the fiber. If the pulse frequency is equal to the gain, the pulses are delayed and if it is equal to the loss, they are accelerated. Thereby, the pulse power, the pump power and hence the time delay can be controlled by tunable optical attenuators or EDFAs which are not shown here. Finally, the delayed or accelerated pulses are detected by a photodiode (PD) on port 3 of the circulator and interpreted via an oscilloscope (Osci). A typical result of delayed pulses is shown on the right hand side of Fig. 3.

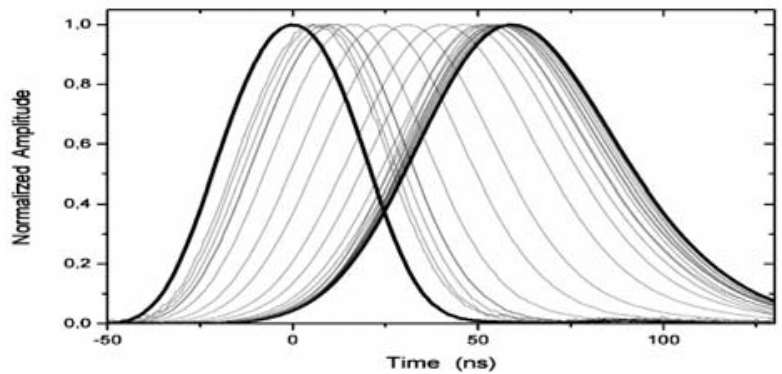

Fig. 3 Principle experimental setup and delayed pulses. 


\section{Limits of the method}

The SBS also has several disadvantages. First, the bandwidth of the SBS in a SSMF is with around $30 \mathrm{MHz}$ very narrow. On the one hand, this limits the maximum delayable data rate drastically. On the other hand, high time delays can be achieved as can be seen in Eq. (6). But, this time delay is accompanied by pulse distortions (pulse shape broadening) due to the spectral narrowing by the SBS gain and the group velocity dispersion (GVD) with higher orders of Eq. (4). The pulse broadening factor $B$ as the relation between the output $-\tau_{\text {out }}$ and input pulse width $\tau_{\text {in }}$ can be described by [20]:

$$
B=\frac{\tau_{\text {out }}}{\tau_{\text {in }}}=\sqrt{1+\frac{16 \ln 2}{\tau_{\text {in }}^{2} \gamma_{1}^{2}} g_{1}} .
$$

Second, the maximum time delay is limited due to the saturation of the Brillouin amplification process (pump depletion) with increasing pump powers. In most of the Slow-Light systems the maximum time delay is around $30 \mathrm{~ns}$ by using SBS with the natural bandwidth. Hence, different mechanisms for tailoring the shape of the Brillouin spectrum have been investigated.

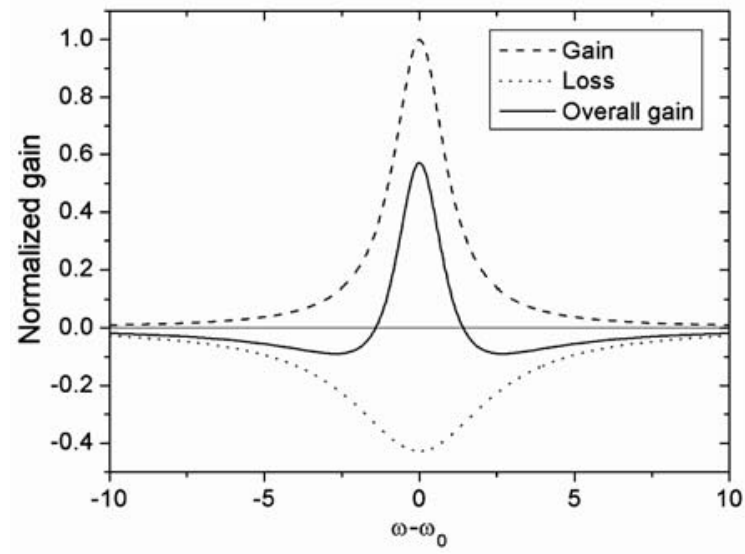

Fig. 4 Superposition of a Brillouin gain with a broadened loss (left) and two losses at its wings (right)

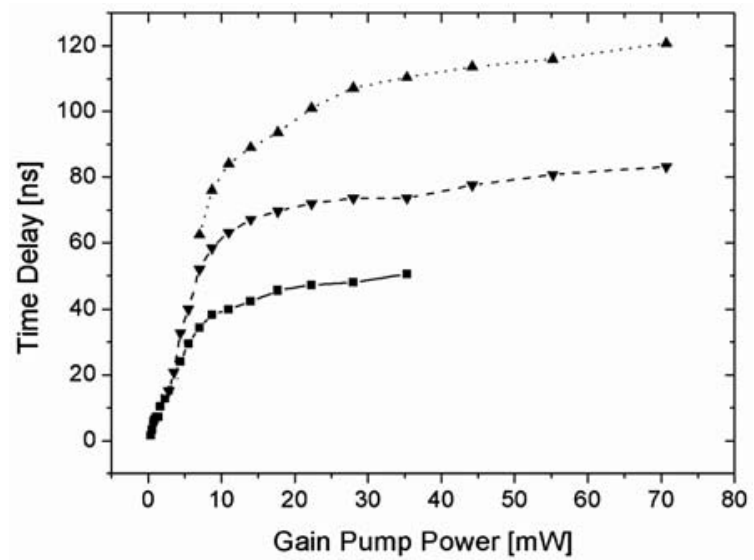

The natural Brillouin bandwidth $\Delta f_{B}$ is defined by parameters of the used fiber. But, the resulting bandwidth $\gamma$ is based on a convolution of the bandwidth of the pump signal $\Delta f_{P}$ and $\Delta f_{B}[17]$ :

$$
\gamma=\pi\left(\Delta f_{P} \otimes \Delta f_{B}\right)
$$

Hence, the Brillouin bandwidth can easily be broadened by direct modulation of the pump source with a noise signal for instance [21]. If only one gain pump source is used this provides a bandwidths up to the value of $f_{B}[22]$. We were able to show that it can be further enhanced to more than $10 \mathrm{GHz}$ by using multiple gain pump sources [23]. A broad gain provides higher delayable data rates and according to Eq. (7) it compensates the pulse distortions partially because the gain does not restrict the spectral distribution of the pulse and the top of the spectrum becomes uniform [24]. However, this decreases the time delay significantly, as can be seen from Eq. (6).

An enhancement of the maximum time delay can be achieved by a superposition of Brillouin gain and loss spectra which decouples the time delay from the amplifier gain. Therefore the setup in
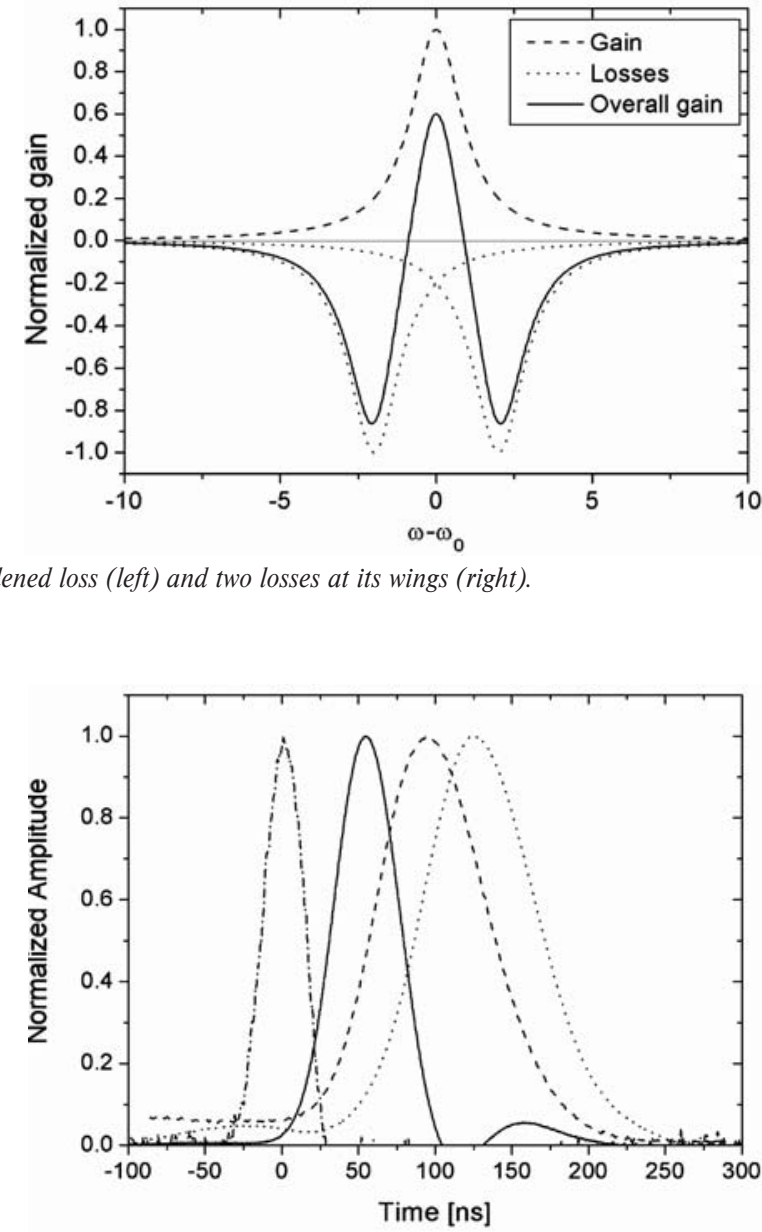

Fig. 5 Time delay vs. gain pump power (left) and pulse time functions with the maximum time delay (right) for a normal gain (solid), a gain superimposed with a broadened loss (dashed) and superimposed with two losses at its wings (dotted); reference (dashed-dotted). 
Fig. 3 has to be extended with further pump sources. Two superposition methods and some measurement results are shown in Fig. 4 and Fig. 5.

A simple method for a realization of the time delay enhancement is to superimpose the Brillouin gain with a broadened Brillouin loss (left diagram of Fig. 4) [25], [26]. The delay depends on the gradient of the gain whereas the amplification and the saturation respectively is a function of the maximum height of the gain. With the superposition of the Brillouin spectrum the gradient is not changed but the height is reduced. Hence, higher pump powers are possible which leads to a higher time delay. With this method we were able to achieve time delays up to $100 \mathrm{~ns}$ which corresponds to around three times the initial pulse width. But, due to a doubling of the pulse width the effective time delay - the ratio between the time delay and the output pulse width - was decreased (dashed lines in Fig. 5).

A method which is more effective is the superposition of a Brillouin gain with two losses at its wings (right diagram of Fig. 4) because this enables even higher time delays because the gradient of the gain is changed in addition to the gain-delaydecoupling. Furthermore, a designing of the Brillouin spectrum by varying the separation of the losses is possible. For this, the complex wave number can be written as [19]: tailoring of the Brillouin spectrum can be done by a variation of different parameters. At first, the time delay and the bandwidth depends directly on $\delta$. Furthermore, the ratio between pump powers $g_{2} / g_{1}$ and the ratio between the bandwidths $\gamma_{2} / \gamma_{1}$ of the losses and the gain have a large influence. Hence, different operating points with different properties can be adjusted [27], as can be seen in Fig. 6.

For $g_{1}=g_{2}$ we determined the region there the maximum time delay can be achieved at $\delta \approx \gamma \sqrt{3}$. In this case it makes no difference if the gain is broadened or not. For equal bandwidths the optimal points of the highest bandwidth (lowest distortions) and so the highest effective time delay occurs at the same position (left diagram of Fig. 6). But, if the gain bandwidth is broadened here to three times of the loss bandwidth - the region of the maximum bandwidth and so the maximum effective time delay is shifted to a higher value of $\delta=2.25 \gamma$ (right diagram of Fig. 6).

With equal gain and loss bandwidths and a loss separation of $\delta=\gamma \sqrt{3}$ we were able to enhance the maximum time delay to around 120 ns which corresponds to four times the initial pulse width [19]. But, the pulse distortion is still high which reduces the effective time delay again (dotted lines in Fig. 5).

To reduce the distortions the gain spectrum can be broadened additionally to get a broad and steep Brillouin profile with a flat

$$
k(\omega)=\frac{n_{0}}{c} \omega+\frac{g_{1}}{z}\left(\frac{\gamma_{1}}{\left(\omega-\omega_{0}\right)+j \gamma_{1}}\right)-\frac{g_{2}}{z}\left(\frac{\gamma_{2}}{\left(\omega-\left(\omega_{0} \pm \delta\right)\right)+j \gamma_{2}}\right),
$$

with the Brillouin coefficient $g_{2}$, the HWHM-bandwidth $\gamma_{2}$ and with the separation of the losses $2 \delta$. With this configuration the top. Then, the gain becomes Gaussian shaped and the complex wave number changes to [19]:

$$
k(\omega)=\frac{n_{c}}{c} \omega+\frac{g_{1}}{j z}\left(e^{-\left(\frac{\omega-\omega_{0}}{\gamma_{G}}\right)^{2}} \cdot \operatorname{erfc}\left(-j \frac{\omega-\omega_{0}}{\gamma_{0}}\right)\right)-\frac{g_{2}}{z}\left(\frac{\gamma_{2}}{\left(\omega-\left(\omega_{0} \pm \delta\right)\right)+j \gamma_{2}}\right),
$$
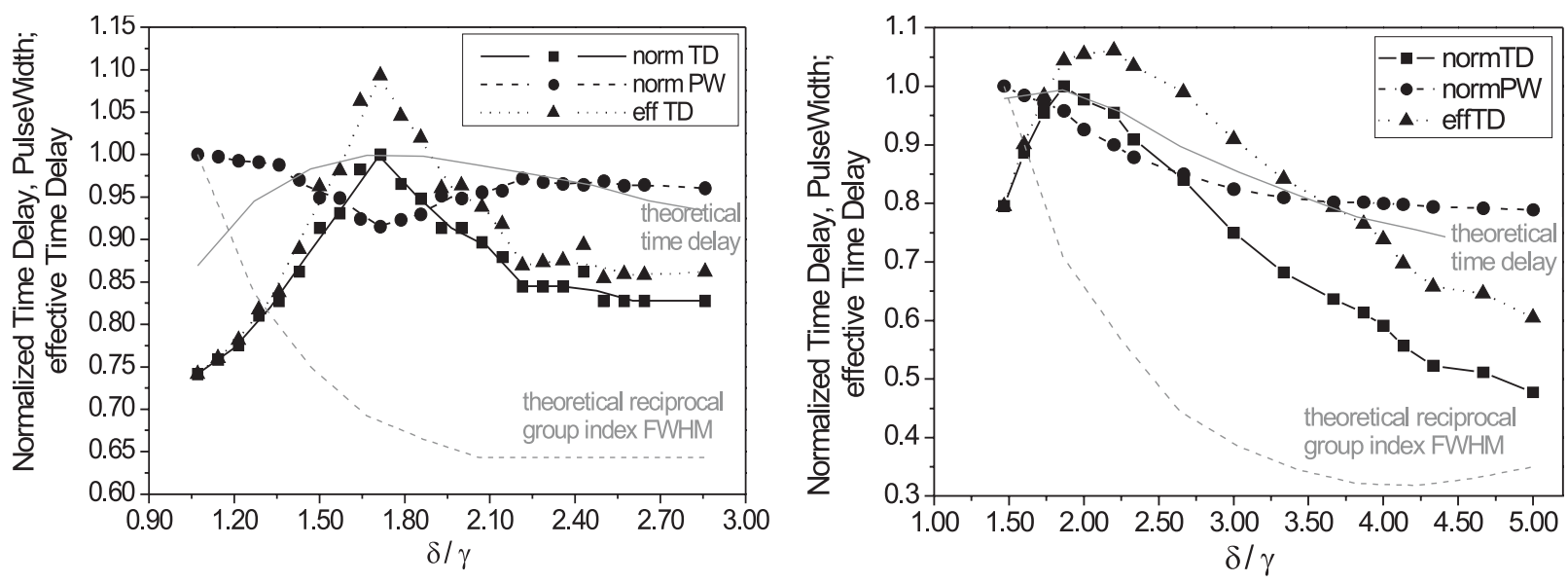

Fig. 6. Normalized time delay (normTD), pulse width (normPW) and effective time delay (effTD) vs. $\delta$ for a natural (left, $\left.\gamma_{1}=\gamma_{2}\right)$ and a broadened (right, $\gamma_{G}=3 \gamma_{2}$ ) Brillouin gain. 

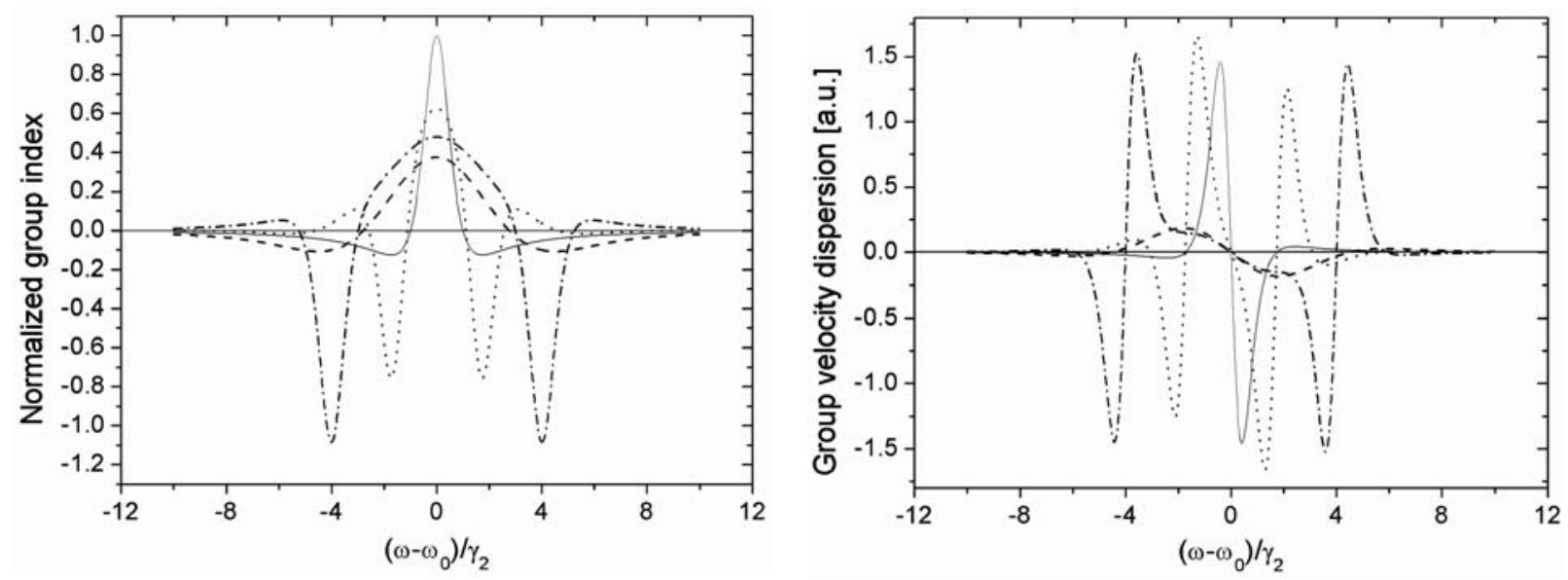

Fig. 7. Normalized group index (left) and GVD (right) for a natural gain (solid) and three times broadened gain (dashed) with losses at $\delta= \pm 1.7 \gamma_{2}$ (dotted) and $\delta= \pm 4 \gamma_{2}$ (dashed-dotted).

with $\gamma_{G}$ as the $1 /$ e-bandwidth of the gain and $\operatorname{erfc}()$ as the complementary error function. Simulations have been shown that a reduction of the GVD next to the bandwidth-broadening is possible with this system [28]. In Fig. 7 it is shown that by a broadening of the gain and a superposition with two losses at its wings the group index and so the time delay is indeed decreased, but the GVD runs linearly with a very small slope in the middle of the Brillouin profile. Therefore, the two operating points for the maximum time delay or the maximum bandwidth could be chosen. In comparison to that, a single natural gain would provide a higher time delay but also a higher GVD and a single broadened gain would provide a lower GVD but at the same time a lower time delay. With a gain bandwidth broadened by the factor of two and a loss separation of $60 \mathrm{MHz}$ we achieved a distortion reduction of $23 \%$ for a delay of one initial pulse width [29].

Nevertheless, the broadening is still a limiting factor which decreases the storage capability and the quality of the Slow-Light system. Recently, it has been shown theoretically that a pulse delay without a broadening could be produced by another method consisting of a broadband gain with a narrow loss in its line center [30]. This is a very important achievement because any desired time delay would be possible without a distortion by connect several delay lines in series. But, due to the broad gain the time delay of any stage would be very small and further decreased by the loss, so that a lot of segments would be necessary and the system would become very complex.

In [31] we have shown in theory and for the first time, to the best of our knowledge, experimentally that a zero-broadening and even a compression of delayed pulses are possible with our system as described in [19] and [29]. Therefore, Gaussian shaped input pulses with a temporal width $\tau_{\text {in }}$ of $1.5 \mathrm{~ns}$ were delayed to approximately $1.5 \mathrm{Bit}$ in a SSMF with a length of $25 \mathrm{~km}$. The non-delayed output pulses had a width $\tau_{\text {ref }}$ of 1.9 ns owing to the fiber dispersion. By an optimization of the separation of the two losses and the loss-gain-ratio it was possible to compress the delayed pulse to $80 \%$ of the reference pulse width, as can be seen in Fig. 8. Hence, the output pulse width comes very close to that for the input pulse width. However, this comes at the expense of a new pulse shape distortion where a small part separates from the main pulse and forms an additional one. But, this part is not as large as the main pulse. Furthermore, we believe that it can be prevented by further optimizations.

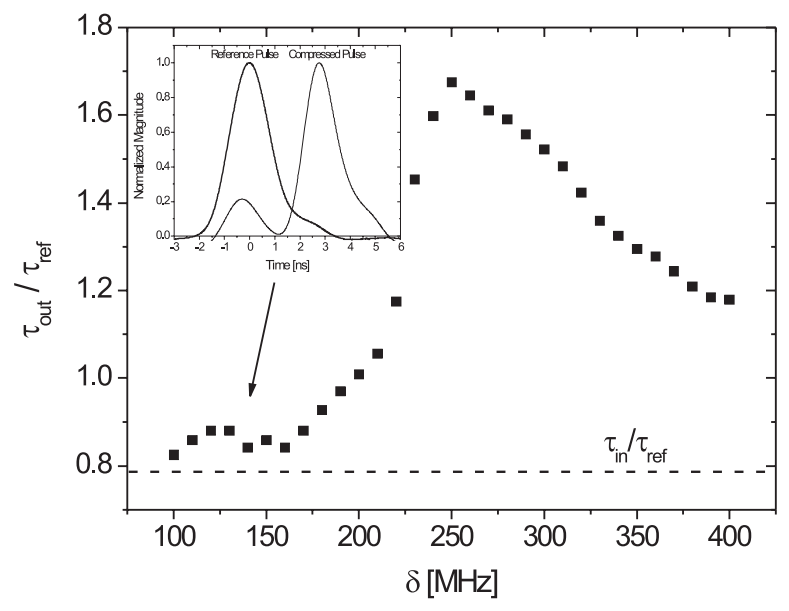

Fig. 8. Fractional output pulse width as a function of the loss separation; the inset shows the delayed compressed pulse in comparison to the non-delayed reference pulse.

How much the effective time delay of these procedures is limited theoretically is not really known at this moment. In [32] it is supposed that the maximum time delay - for a maximum tolerable pulse broadening factor of $B=2$ - is:

$$
\left(\frac{\Delta T_{S B S}}{\tau_{i n}}\right)_{\max }=\frac{3}{2} \gamma \tau_{i n} .
$$

By choosing $\gamma$ arbitrarily the effective time delay could reach any dimension. Another assumption is shown in [33] where the gain $G$ for buffering $N$ Bits has to be: 


$$
G \approx 50 N^{\frac{3}{2}}
$$

For a storage of more than 5 Bits the gain should be higher than $560 \mathrm{~dB}$ which is not realistic. Even for a 3 Bit delay $260 \mathrm{~dB}$ would be needed. But, we used a gain of only $16 \mathrm{~dB}$ [26]. Hence, in Eq. (12) there is disregarded the opportunity to decouple the time delay and the gain.

In general, the achievable delay in SBS-based Slow-Light systems depends on the saturation gain and therefore on the input pulse power. For our system of a superposition of a Brillouin gain with two losses at its wings an enhancement of the time delay by a factor of 2 up to 9.5 could be achieved for input pulse powers of $-60 \mathrm{dBm}$ and $0 \mathrm{dBm}[34]$.

\section{Conclusions}

In this paper an overview about the fundamentals, the limits and achieved experimental results of Slow- and Fast-Light systems based on SBS was given. The existing experimental setups of such systems are still far away from real practical applications and the final limits are still unknown nowadays. But, there are reinforced investigations on this topic worldwide. Next to the fundamental interest on the control of the light speed in media Slow- and FastLight can be seen as a key technology for future packet switched optical networks.

\section{Acknowledgements}

We gratefully acknowledge the help of J. Klinger from HfT Leipzig and the loan of the equipment of C. Schaeffer from TUDresden and of K. Buse from the University in Bonn. M. Junker, K.-U. Lauterbach and R. Henker were financially supported by Deutsche Telekom.

\section{References}

[1] BERNASCONI, P.; SIMSARIAN, J. E.; GRIPP, J.; DULK, M.; NEILSON, D. T.: Toward Optical Packet Switching, Photonics Spectra, 03/2006, pp. 4-92.

[2] SCHNEIDER, T.; HENKER, R.; LAUTERBACH, K.-U.; JUNKER, M.: Slow und Fast Light in photonischen Netzen - ein Überblick, Proc. 8. ITG-Fachtagung Photonische Netze, vol. 201, 2007, pp. 17-24.

[3] Journ. of Lightw. Technol. (Special Issue on Optical Networks), vol. 18, 12/2000.

[4] NAGATSU, N.: Photonic Network Design Issues and Applications to the IP Backbone, Journ. of Lightw. Technol., vol. 18, no. 12, 12/2000, pp. 2010-2018.

[5] CHANG-HASNIAN, C. J.; KU, P.-C.; KIM, J.; CHUANG, S.-L.: Variable Optical Buffer Using Slow Light in Semiconductor Nanostructures, Proc. IEEE, vol. 91, 2003, pp. 1884-1897.

[6] HAU, L. V.; HARRIS, S. E.; DUTTON, Z.; BEHROOZI, C. H.: Light speed reduction to $17 \mathrm{~m} / \mathrm{s}$ in an ultracold atomic gas, Nature, vol. 397, 1999, pp. 594-598.

[7] KASH, M. M.; SAUTENKOV, V. A.; ZIBROV, A. S.; HOLLBERG, L.; WELCH, G. R.; LUKIN, M. D.; ROSTOVTSEV, Y.; FRY, E. S.; SCULLY, M. O.: Ultraslow group velocity and enhanced nonlinear optical effects in a coherently driven hot atomic gas, Phys. Rev. Lett., vol. 82, 06/1999, pp. 5229-5232.

[8] KU, P.-C.; SEDGWICK, F.; CHANG-HASNAIN, C. J.; PALINGINIS, P.; LI, T.; WANG, H.; CHANG, S.-W.; CHUANG, S.-L.: Slow light in semiconductor quantum wells, Opt. Lett., vol. 29, 2004, pp. 2291-2293.

[9] POEL, M. van der; MORK, J.; HVAM, J.: Controllable delay of ultrashort pulses in a quantum dot optical amplifier, Opt. Express, vol. 13, 2005, pp. 8032-8037.

[10] GERSEN, H.; KARLE, T. J.; ENGELEN, R. J. P.; BOGAERTS, W.; KORTERIK, J. P.; HULST, N. F. VAN; KRAUSS, T. F.; KUIPERS, L.: Real-space observation of ultraslow light in photonic crystal waveguides, Physical Review Letters, vol. 94, 2005, 073903.

[11] LEPESHKIN, N. N.; SCHWEINSBERG, A.; BIGELOW, M. S.; GEHRING, G.; BOYD, R. W.: Slow and fast light propagation in erbium-doped fiber, Quantum Electronics and Laser Science Conference (QELS'05), vol. 1, 05/2005, pp. 220-222.

[12] USKOV, A. V.; SEDGWICK, F. G.; CHANG-HASNAIN, C. J.: Delay limit of slow light in semiconductor optical amplifiers, IEEE Photonics Technology Letters, vol. 18, 03/2006, pp. 731-733.

[13] SHARPING, J. E.; OKAWACHI, Y.; HOWE, J. VAN; XU, C.; GAETA, A. L.: All-optical, continuously tunable, nanosecond pulse delay using wavelength conversion and fiber dispersion, Conference on Lasers and Electro-Optics (CLEO), vol. 2, 05/2005, pp. 971-973.

[14] SHARPING, J. E.; OKAWACHI, Y.; GAETA, A. L.: Wide bandwidth slow light using a Raman fiber amplifier, Opt. Express, vol. 13, 2005, pp. 6092-6098.

[15] OKAWACHI, Y.; BIGELOW, M. S.; SHARPING, J. E.; ZHU, Z. M.; SCHWEINSBERG, A.; GAUTHIER, D. J.; BOYD, R. W.; GAETA, A. L.: Tunable all-optical delays via Brillouin slow light in an optical fiber, Phys. Rev. Lett., vol. 94, 2005, 153902.

[16] GONZALEZ-HERRAEZ, M.; SONG, K.-Y.; THAVENAZ, L.: Optically controlled slow and fast light in optical fibers using stimulated Brillouin scattering, Applied Physics Letters, vol. 87, 2005, 081113. 
[17] SCHNEIDER, T.: Nonlinear Optics in Telecommunications, Springer-Verlag, Berlin, 2004.

[18] STENNER, M. D.; NEIFELD, M. A.; ZHU, Z.; DAWES, A. M. C.; GAUTHIER, D. J.: Distortion management in slow-light pulse delay, Opt. Express, vol. 13, 2005, pp. 9995-10002.

[19] SCHNEIDER, T.; HENKER, R.; LAUTERBACH, K.-U.; JUNKER, M.: Comparison of delay enhancement mechanisms for SBSbased slow light systems, Opt. Express, vol. 15, 2007, pp. 9606-9613.

[20] ZHU, Z.; GAUTHIER, D. J.; OKAWACHI, Y.; SHARPING, J. E.; GAETA, A. L.; BOYD, R. W.; WILLNER, A. E.: Numerical Study of All-Optical Slow-Light Delays via Stimulated Brillouin Scattering in an Optical Fiber, J. Opt. Soc. Am. B, vol. 22, 2005, pp. 2378-2384.

[21] GONZALEZ-HERRAEZ, M.; SONG, K. Y.; THEVENAZ, L.: Arbitrary-Bandwidth Brillouin Slow Light in Optical Fibers, Opt. Exp., vol. 14, 02/2006, pp. 1395-1400.

[22] ZHU, Z.; DAWES, A. M. C.; GAUTHIER, D. J.; ZHANG, L.; WILLNER, A. E.: Broadband SBS slow light in an optical fiber, J. Lightwave Technol., vol. 25, 2007, pp. 201-206.

[23] SCHNEIDER, T.; JUNKER, M.; LAUTERBACH, K.-U.: Potential ultra wide slow-light bandwidth enhancement. Opt. Express, vol. 14, 2006, pp. 11082-11087.

[24] PANT, R.; STENNER, M. D.; NEIFELD, M. A.: Designing Optimal Gain Profiles for Slow-Light Applications, Proc. of SPIE, vol. 6482, 2007, 64820R

[25] CHIN, S.; GONZALEZ-HERRAEZ, M.; THÉVENAZ, L.: Zero-gain slow \& fast light propagation in an optical fiber, Opt. Express, vol. 14, 2006, pp. 10684-10692.

[26] SCHNEIDER, T.; JUNKER, M.; LAUTERBACH, K.-U.: Time delay enhancement in stimulated Brillouin-scattering-based slow-light systems, Opt. Lett., vol. 32, 2007, pp. 220-222.

[27] HENKER, R.; WIATREK, A.; LAUTERBACH, K.-U.; SCHNEIDER, T.; AMMANN, M. J.; SCHWARZBACHER, A. T.: Optimization of the Brillouin spectrum for fiber based slow light systems, Proc. of CLEO'08, 2008, CThE1.

[28] HENKER, R.; WIATREK, A.; LAUTERBACH, K.-U.; AMMANN, M. J.; SCHWARZBACHER, A. T.; SCHNEIDER, T.: Group velocity dispersion reduction in fibre-based slow-light systems via stimulated Brillouin scattering, Electronics Letters, vol. 44, 2008, pp. $1185-1187$.

[29] SCHNEIDER, T.; HENKER, R.; LAUTERBACH, K.-U.; JUNKER, M.: Distortion reduction in Slow Light systems based on stimulated Brillouin scattering, Opt. Express, vol. 16, 2008, pp. 8280-8285.

[30] WANG, S.; REN, L.; LIU, Y.; TOMOTA, Y.: Zero-broadening SBS slow light propagation in an optical fiber using two broadband pump beams, Opt. express, vol. 16, 2008, pp. 8067-8076.

[31] SCHNEIDER, T.; WIATREK, A.; HENKER, R.: Zero-broadening and pulse compression slow light in an optical fiber at high pulse delays, Opt. Express, vol. 16, 2008, pp. 15617-15622.

[32] BOYD, R. W.; GAUTHIER, D. J.; GAETA, A. L.; WILLNER, A. E.: Limits on the Time Delay Induced by Slow-Light Propagation, Proc. of QELS'05, 2005, QTuC1.

[33] KHURGIN, J. B.: Performance Limits of Delay Lines Based on Optical Amplifiers, Opt. Lett., vol. 31, 2006, pp. $948-950$.

[34] SCHNEIDER, T.: Time delay limits of stimulated-Brillouin-scattering-based slow light systems, Opt. Letters, vol. 33, 2008, pp. $1398-1400$. 